\title{
Über die Veränderlichkeit systematisch wichtiger Merkmale, nebst Bemerkungen zu den Gattungen Thrips und Euthrips.
}

Von H. Karny in Wien.

(Mit 10 Figuren im Texte)

Es ist eine bemerkenswerte Tatsache und wird vielleicht $\mathrm{zu}$ wenig beachtet, daf jene Merkmale, die wir im allgemeinen als gute Art- und Gattungsunterschiede und somit für die Systematik bedeutungsvoll und wichtig ansehen, auch mitunter gewissen Schwankungen unterworfen sind. Ich möchte hier in erster Linie auf das Flügelgeäder der Insekten hinweisen. Es ist bekannt, daf dessen Verwertung in der Systematik sich als außerordentlich fruchtbar erwiesen hat und namentlich jüngst haben die bahnbrechenden Untersuchungen von A. Handlirsch über ,die fossilen Insekten und die Phylogenie der rezenten Formen" dies wieder in weittragender und großartiger Weise bestätigt.

Und doch können wir wieder einzelne Fälle konstatieren, wo sich hierin Aberrationen vom Typus vorfinden. So habe ich seinerzeit auf "eine Mißbildung des Hinterflügels bei Psophus stridulus" hingewiesen und ebenso ist jedem Orthopterologen bekannt, welch auffallenden Variationen das Geäder der Gryllacriden unterworfen ist. Gelegentlich der Bearbeitung der "Blattaeformia Oothecaria" von Südwestafrika (Jenaische Denkschriften 1908) habe icheinen anomalen Flügel meiner Ischnoptera uniramosa abgebildet und eine Geädervarietät von Blattella germanica beschrieben (Var. Shuguroffi m.). Auch bei Gonypeta wurde auf die Inkonstanz des Geäders hingewiesen (1. c.).

Diesmal will ich Beispiele aus anderen Insektenordnungen vorbringen. So ist unter den Cicadinen, namentlich bei den Typhlocyben, das Flügelgeäder für die Gattungsunterscheidung sehr wichtig. Ich besitze nun eine Alebra albostriella aus Pötzleinsdorf, bei welcher der linke Vorderflügel normal gebildet ist, während der rechte an der Basis der Endzellen zwei kleine Zellen besitzt, die dem normalen Flügel fehlen, und zwar eine am Vorderrande und eine ungefähr in der Mitte der Flügelbreite (Fig. 1, 2.).

Noch wesentlicher ist eine andere Variation: Für die Gattung Athysanus ist es bekanntlich charakteristisch, daß die Media nur

Wiener Entomologische Zeitung, XXVII. Jahrg., Heft IX und X (1. Dezember 1908). 
knapp nach ihrer Trennung vom Radius (= erste Gabelung des „Sector primus“) mit dem Cubitus durch eine Querader verbunden ist. Dann ist keine Querader mehr vorhanden bis knapp vor der Spitze bei den Endzellen. Dagegen besitzen z. B. Deltocephalus

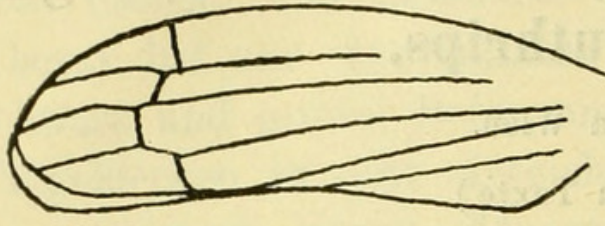

Fig. 1. Normaler Vorderflügel einer Alebra albostriella. Original (c. m.) 10 fach vergr.

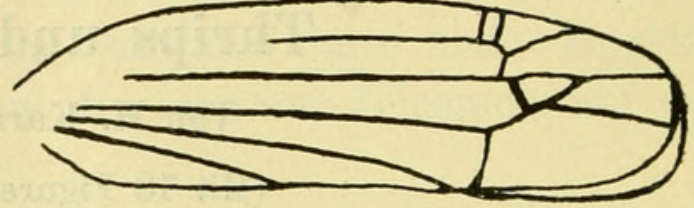

Fig. 2. Abnormer Vorderflügel von Alebra albostriella. Original (c. m.). 10 fach vergr.

und Jassus (= Allygus) auch noch eine oder zwei Queradern bei der Gabelung der Media (= zweite Gabelung des "Sector primus“). Die Spezies Athysanus procerus nun ist am Eichkogel bei Mödling ziemlich häufig und ich habe eine Anzahl Exemplare dort gesammelt. Bei diesen zeigte sich nun, daß oft auch bei der Gabelung der Media eine oder zwei Queradern vorhanden waren, wie dies doch bei einem Athysanus nicht der Fall sein sollte. Und zwar bemerkte ich, daß hier gewöhnlich beide Vorderflügel gleich gebildet waren, entweder beide nach dem Typus Athysarus oder beide nach dem Typus Deltocephalus. Und doch stimmten alle Exemplare im übrigen vollkommen miteinander überein und gehören bestimmt zur selben Art (Fig. 3, 4).

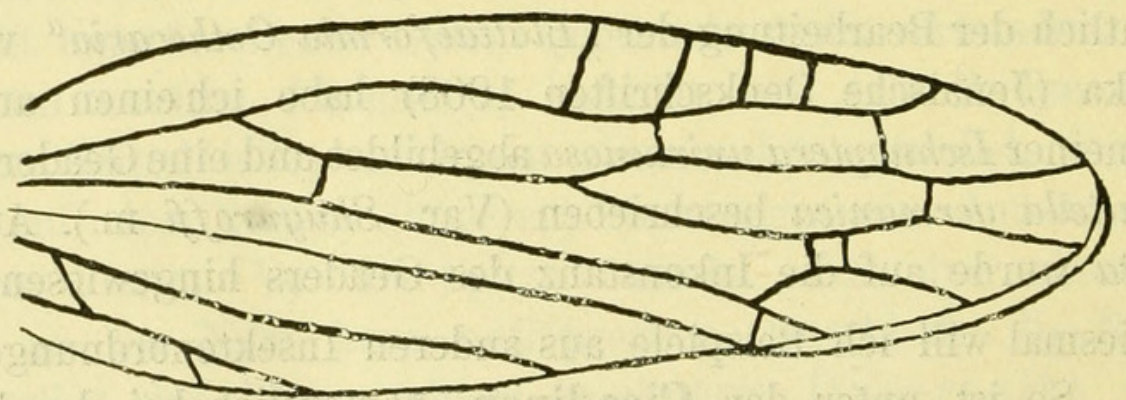

Fig. 3. Vorderflügel von Athysanus procerus, typische Form. Original (c. m.). 10fach vergr.

Nun ein paar Beispiele aus der Gruppe der Copeognathen (Psocoidea). Zunächst ein Fall ähnlich dem von Alebra. Ein Caecilius fuscopterus vom Bisamberg, der sich in meiner Sammlung befindet, zeigt links am Vorder- und Hinterflügel um je eine kleine Zelle mehr, als er normalerweise besitzen sollte. Rechts sind Vorder- 
und Hinterflügel normal. Ich bilde alle vier Flügel des Exemplares ab (Fig. 5).

Überhaupt scheint gerade bei der Gattung Caecilius das Geäder etwas variabel zu sein. Bei den Caeciliinen soll doch ganz allgemein die Diskoidalzelle (= Zelle zwischen Media und Cubitus) offen sein,

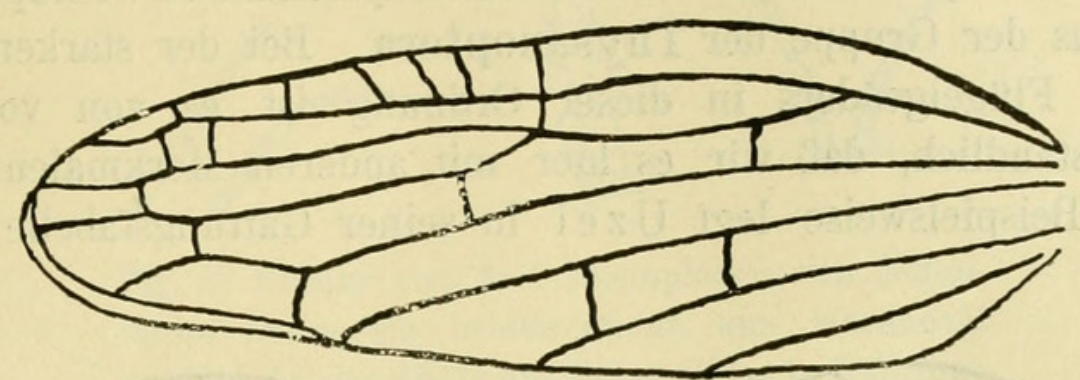

Fig. 4. Vorderflügel von Athysanus procerus var. nov. jassoides m. Original (c. m.). 10fach vergr.
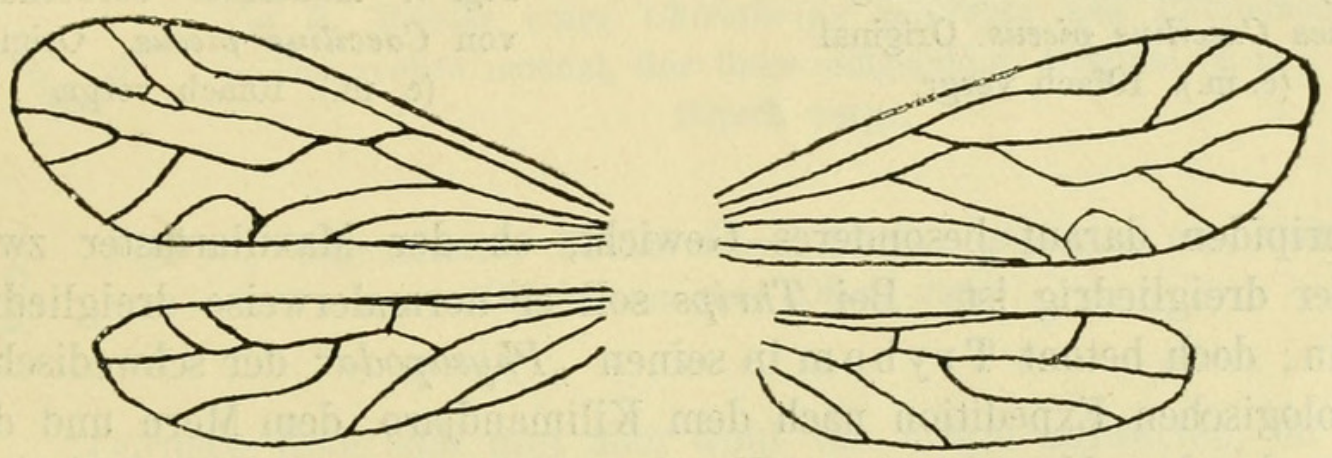

Fig. 5. Alle vier Flügel eines Caecilius fuscopterus.

Original (c. m.). 10fach vergr.

während die Stenopsocini und Psocini durch eine geschlossene Diskoidalzelle charakterisiert sind. Ich besitze nun einen Caecilius flaidus vom Bisamberg und einen Caecilius piceus vom Anninger, welche an dem einen Vorderflügel eine offene (normale), am anderen eine geschlossene Diskoidalzelle haben, indem hier die Umgrenzungsader der Hinterrandzelle (= distaler Teil des Cubitus) durch eine Querader mit der Media verbunden ist (Fig. 6, 7), wie es sonst nur bei den Stenopsocinen vorkommt. Doch wären derartige Exemplare immerhin noch als Caeciliini zu erkennen, auch wenn die Variation an beiden Flügeln vorhanden wäre (ein solcher Fall ist mir jedoch bisher nicht bekannt). Denn bei den Psocinen kommt die Schließung der Diskoidalzelle nicht durch eine Querader zu stande, sondern dadurch, daß Cubitus und Media eine kurze Strecke weit 
miteinander verschmelzen. Bei den Stenopsocinen und abnormen Caeciliinen ist jedoch keine Verschmelzung, sondern eine Querader vorhanden und diese beiden Gruppen sind voneinander wieder daran leicht zu unterscheiden, daß bei den ersteren unter dem Pterostigma eine Querader zum Radii Sector abgeht, die den Caeciliinen stets fehlt.

Nun Beispiele der „Veränderlichkeit systematisch wichtiger Merkmale" aus der Gruppe der Thysanoptera. Bei der starken Reduktion des Flügelgeäders in dieser Ordnung ist es von vornherein selbstverständlich, daß wir es hier mit anderen Merkmalen zu tun haben. Beispielsweise legt Uzel in seiner Gattungstabelle bei den

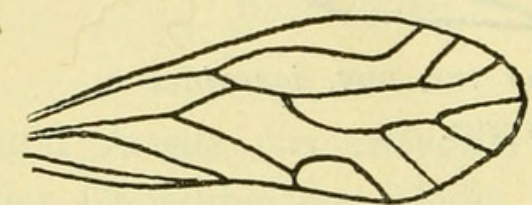

Fig. 6. Normaler Vorderflügel eines Caecilius piceus. Original (c. m.). 10 fach vergr.

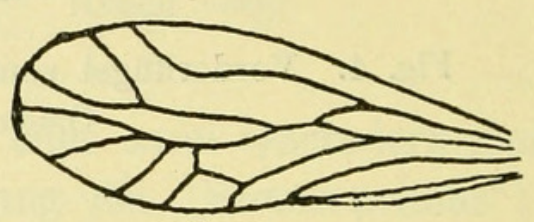

Fig. 7. Abnormer Vorderflügel von Caecilius piceus. Original (c. m.). 10fach vergr.

Thripiden darauf besonderes Gewicht, ob der Maxillartaster zweioder dreigliedrig ist. Bei Thrips soll er normalerweise dreigliedrig sein; doch betont Trybom in seinen "Physapoda" der schwedischen zoologischen Expedition nach dem Kilimandjaro, dem Meru und den umgebenden Massaisteppen (Upsala 1908), daß er „unter lichtgefärbten Männchen von Thrips physapus $L$. aus Schweden einige gesehen habe, an deren Maxillartastern er „keine Grenze zwischen den beiden äußeren Gliedern wahrnehmen konnte" (l. c. pag. 10).

Ein Merkmal, auf welches in der Speziessystematik viel Wert gelegt wird, ist die Zahl der Borsten auf dem distalen Teile der Hauptader. Thrips communis besitzt deren vier und von ihr unterscheidet sich Thrips flava nach Uzel (pag. 188) „durch immer nur drei Borsten in der zweiten Hälfte der Hauptader". Ich habe in Aflenz (Hochschwabgebiet) in Spiraea-Blüten ein Exemplar von Thrips flava gesammelt, das auf dem distalen Teile der Hauptader auf dem einen Vorderflügel drei, auf dem anderen fünf Borsten hat.

Von der allergrößten Bedeutung in der Thysanopteren-Systematik ist Zahl und Form der Fühlerglieder. Und doch läßt sich ja von vornherein voraussetzen, daß hier durch Verletzung und Regeneration der Variabilität der weiteste Spielraum geboten ist. Ganz dasselbe finden wir ja auch bei den Poduriden, in deren Monographie 
ja schon Lubbock einige Exemplare mit abnormen, regenerierten Fühlern abgebildet hat. Statt aller eingehenden Erörterungen gebe ich umstehend Abbildungen abnormer Thysanopterenfühler nach Originalen meiner Sammlung (Fig. 8 bis 10).
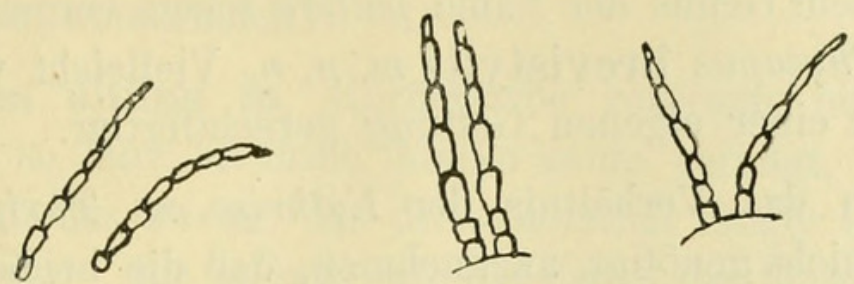

Fig. 8. Fühler von drei Exemplaren von Melanthrips fusca (die beiden ersten aus Maranovič auf Meleda, das dritte aus Przywalicha in Galizien). Nur der erste Fühler normal, die übrigen mit weniger als der normalen Gliederzahl. Original

(c. m.). 30fach vergr.

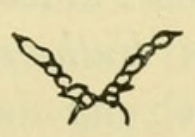

Fig. 9. Fühler einer Chirothrips manicata aus Pötzleinsdorf. Der rechte normal, der linke mißgebildet. Original (c. m.).

30 fach vergr.

Fig. 10. Regenerierter Fühler einer Thrips physapus aus Cattaro.
Original (c. m.). 30fach vergr.

Darnach ließe sich nun aber wohl die Frage aufwerfen, ob die fundamentale Scheidung, die Uzel zwischen Thripiden (s. str.) mit acht- und solchen mit siebengliedrigen Fühlern macht, gerechtfertigt ist oder, um spezieller zu sprechen, ob beispielsweise die Trennung der Gattungen Thrips und Euthrips wirklich aufrechterhalten werden kann. Ich habe auf diesen Punkt schon einmal (Mitt. Naturw. Ver. Univ. Wien, 1908, pag. 111) mit ein paar Worten hingewiesen und will hier meinen Standpunkt in dieser Frage eingehender darlegen.

Vor allem muß ich betonen, daß meiner Ansicht nach Euthrips der Gattung Thrips viel näher steht als der Gattung Physapus; ich finde in dem Vorhandensein oder Fehlen der Borsten an den Vorderecken des Prothorax ein viel wesentlicheres Merkmal als in der Trennung oder Verschmelzung der beiden Stylusglieder. Daher habe ich schon in meiner „Orthopterenfauna des Küstengebietes von Österreich-Ungarn“ (Berlin. Entom. Zeitschr. 1907, pag. 45) die Trennung der Euthrips von Physapus betont und demgemäß Physapus Uzel nec. Serv. in fünf Gattungen aufgeteilt. Von diesen steht Euthrips

Wiener Entomologische Zeitung, XXVI. Jahrg., Heft IX und X (1. Dezember 1908). 
dem Genus Thrips jedenfalls außerordentlich nahe, während die vier anderen Gattungen mit Thrips gar nichts zu tun haben. Dabei muß ich allerdings bemerken, daß ich meine Thrips pallida (l. c. pag. 49), die auch an den Vorderecken des Prothorax je eine lange Borste besitzt, nunmehr trotz des eingliedrigen Stylus zu Physapus stelle, und da in diesem Genus der Name pallida schon vergeben ist, nenne ich sie jetzt Physapus brevistylis $m$. n. n. Vielleicht wäre übrigens die Errichtung einer eigenen Gattung gerechtfertigt.

Was nun das Verhältnis der Euthrips zu Thrips betrifft, so sind wir wohl nicht genötigt, anzunehmen, daß die beiden Genera vor unseren Augen immer ineinander übergehen; denn wenn bei einer Euthrips infolge eines Regenerationsvorganges zufällig einmal ein Fühler einen eingliedrigen Stylus besitzt, so können wir das noch nicht als solchen Übergang ansehen; um so mehr, da ja Regeneration gewöhnlich noch tiefergreifende Veränderungen herbeizuführen pflegt, wie die obigen Figuren gezeigt haben. Immerhin aber kann es keinem Zweifel unterliegen, daß Thrips auf Ahnen mit achtgliedrigen Fühlern zurückgeht, und solche können wir dann nur im Genus Euthrips suchen. Auch spricht gar nichts dafür, daß die Verschmelzung der beiden letzten Glieder nur einmal eingetreten wäre, vielmehr ist die nächstliegende Annahme ohne Zweifel die einer polyphyletischen Erwerbung dieses Merkmals.

So wären wir denn nun vor die Aufgabe gestellt, zu den einLelnen Thrips-Arten ihre Euthrips-Ahnen aufzusuchen. Dabei müssen wir uns aber stets vor Augen halten, daf 1. manche Euthripsarten bis jetzt auf diesem Stadium stehen geblieben und es somit noch nicht zu Thrips-Nachkommen gebracht haben können, und 2. daß zu manchen Thrips-Arten die zugehörigen Euthrips-Ahnen schon ausgestorben sein können. Außerdem müssen wir natürlich auch damit rechnen, daß uns noch viele Arten beider Gattungen unbekannt sind und so wesentliche Bindeglieder fehlen können.

Jedenfalls glaube ich aber, daß der hier vorgeschlagene Standpunkt für die Beurteilung der beiden Gattungen und ihrer Arten in Zukunft fruchtbringend werden dürfte. Doch müssen wir uns vor allem bemühen, reicheres Beobachtungsmaterial zu sammeln. Um auch dazu beizutragen, will ich bei dieser Gelegenheit gleich zwei neue Euthrips-Arten beschreiben, die vielleicht in phylogenetischer Hinsicht interessant sein dürften. Es handelt sich um die beiden Arten Euthrips alpina m. und Euthrips Trybomi m. Ich habe die Beschreibungen der beiden in meinen „Beitrag zur alpinen Ortho- 
pterenfauna" aufgenommen, den ich im August 1907 der Schweizer Entomologischen Gesellschaft für ihre Mitteilungen zusandte. Doch da ich bis heute von dort kein Schreiben, keine Korrektur und keine Separata erhalten habe, muß ich annehmen, daß die Abhandlung in Verlust geraten ist, und sehe mich genötigt, die beiden Neubeschreibungen hier zu wiederholen:

Euthrips alpina m. Körperfarbe rotbraun bis schwärzlich. Kopf ungefähr so lang als breit, hinten kaum verengt. Erstes Fühlerglied kürzer als das zweite, das dritte deutlich länger als das vorhergehende, das vierte fast so lang wie das dritte, das fünfte ist bedeutend kürzer und schmäler als das vierte und legt sich mit mehr oder weniger breiter Fläche an das sechste an. Dieses etwas länger als das dritte. Stylus ziemlich lang, sein zweites Glied länger und schmäler als das erste. Fühlerfärbung dunkelbraun, das dritte Glied gelblich. Prothorax kaum länger als der Kopf, an seinen Hinterecken mit je einer langen Borste (an seinen Vorderecken ohne solche). Alle Beine dunkelbraun. Vorderflügel an der Basis hell, sodann stark braun getrübt. Die Hauptader ist in ihrer zweiten Hälfte mit drei Borsten versehen, von denen die erste von den beiden anderen auffallend weit absteht. Die Nebenader ist ihrer ganzen Länge nach mit Borsten besetzt. Hinterflügel etwas heller als die vorderen. - A flen z (Steiermark), in Blüten von Leontodon (c. m.).

Euthrips Trybomi m. Gelb; der lythri am nächsten stehend. Hauptader in der zweiten Hälfte mit drei Borsten, von denen die erste von den beiden anderen weit entfernt steht. Form der Fühlerglieder ähnlich wie bei alpina. - Ich erlaube mir, die neue Art nach Herrn Dr. A. F. Tr y b o m, dem berühmten schwedischen Thysanopterologen zu benennen, der erst jüngst wieder eine interessante Arbeit über die Kilimandjaro-Physapoden der Öffentlichkeit übergeben hat. - Aflenz, in verschiedenen Blüten.

Die beiden hier beschriebenen Arten sind von allen bisher bekannten Euthrips-Formen gut zu unterscheiden und haben stets einen deutlich zweigliedrigen Stylus; dennoch ließe sich vielleicht an phylogenetische Beziehungen zu Thrips physapus, respektive Thrips flava denken.

Der Zweck der bisherigen Erörterungen soll aber nicht der sein, Thrips und Euthrips zu einer Gattung zu vereinigen. Ich bin zwar von der polyphyletischen Entstehung des Genus Thrips aus Euthrips überzeugt, muß aber dabei betonen, daß die beiden Formengruppen zwei verschiedene Stadien in der phylogenetischen Entwick- 
lung darstellen, die wir immerhin praktisch als konstant nehmen können, um so mehr da die große Artenzahl am einfachsten überblickt werden kann, wenn wir nach wie vor Formen mit siebengliedrigen von denen mit achtgliedrigen Fühlern trennen.

Übrigens werden sich vielleicht auch innerhalb jeder der beiden Gattungen da und dort noch nähere Beziehungen nachweisen lassen. Ich habe oben darauf hingewiesen, daß Thrips flava in der Regel drei, communis vier Borsten auf dem distalen Teile der Hauptader besitzt, daß aber gelegentlich bei flava bis fünf vorkommen. Ähnlich dürften die von mir in der „Orthopterenfauna des Küstengebietes von Österreich-Ungarn" (pag. 45, 46) beschriebenen Euthrips-Arten miteinander näher verwandt sein. Dalmatica erinnert noch ziemlich an atrata (auch was die Borstenzahl betrifft), lythri steht in jeder Beziehung der Trybomi (und der allerdings nicht in Blüten lebenden ulmifoliorum) nahe. Discolor bildet den Übergang von dalmatica zu lythri. Von den gelben Euthrips-Arten hat ulmifoliorum die geringste, lythri die größte Anzahl von Borsten auf dem distalen Teile der Hauptader. Vielleicht existieren übrigens auch Beziehungen zwischen Euthrips lythri und Thrips communis; denn diese beiden Spezies unterscheiden sich im wesentlichen nur durch die Zahl der Fühlerglieder. Vor allem wird es aber notwendig sein, von den dalmatinischen Arten noch mehr Material aufzubringen, da mir alle nur in wenigen Exemplaren vorliegen und diese zum Teil nicht gerade im besten Erhaltungszustande. 


\section{$2 \mathrm{BHL}$ Biodiversity Heritage Library}

Karny, Heinrich Hugo. 1908. "Über die Veränderlichkeit systematisch wichtiger Merkmale, nebst Bemerkungen zu den Gattungen Thrips und Euthrips." Wiener entomologische Zeitung 27, 273-280.

https://doi.org/10.5962/bhl.part.11640.

View This Item Online: https://www.biodiversitylibrary.org/item/43799

DOI: https://doi.org/10.5962/bhl.part.11640

Permalink: https://www.biodiversitylibrary.org/partpdf/11640

\section{Holding Institution}

Smithsonian Libraries

\section{Sponsored by}

Smithsonian

\section{Copyright \& Reuse}

Copyright Status: NOT_IN_COPYRIGHT

This document was created from content at the Biodiversity Heritage Library, the world's largest open access digital library for biodiversity literature and archives. Visit BHL at https://www.biodiversitylibrary.org. 